\title{
Erratum to: Part II: defining and quantifying individual and co-cultured intracellular proteomes of two thermophilic microorganisms by GeLC-MS ${ }^{2}$ and spectral counting
}

\author{
Genna Andrews • Derrick Lewis • Jaspreet Notey • \\ Robert Kelly • David Muddiman
}

Published online: 13 August 2010

(C) Springer-Verlag 2010

\section{Erratum to: Anal Bioanal Chem \\ DOI 10.1007/s00216-010-3929-8}

The corresponding author of this article has let us know that the order of the authors in this article should correctly be Genna Andrews, Derrick Lewis, Jaspreet Notey, Robert Kelly and David Muddiman.

Unfortunately, the following paragraph was missing in the published article:

\section{Supporting Information Available}

Supplemental material for this article (Table S1) and the data associated with this manuscript may be downloaded from ProteomeCommons.org Tranche using the following hash: 8XY7M1CuXZD4mMWws7HcECm2g75k/C5EIBli12qAV Ska6oaFOs9HLN/QjSmnBxZgZgztaz0K4AxQu3yo + 1HhT9xuuTkAAAAAAAArVw==

The hash may be used to prove exactly what files were published as part of this manuscript's data set, and the hash may also be used to check that the data has not changed since publication.

The online version of the original article can be found at http://dx.doi. org/10.1007/s00216-010-3929-8.

\footnotetext{
G. Andrews · D. Muddiman

Chemistry, North Carolina State University,

Raleigh, NC 27695, USA

D. Lewis $\cdot$ J. Notey $\cdot$ R. Kelly

Chemical and Biomolecular Engineering,

North Carolina State University,

Raleigh, NC 27695, USA

D. Muddiman $(\triangle)$

W.M. Keck FT-ICR Mass Spectrometry Laboratory,

North Carolina State University,

Raleigh, NC 27695, USA

e-mail: david_muddiman@ncsu.edu
} 